\title{
Aircraft Trajectory Optimization and Contrails Avoidance in the Presence of Winds
}

\author{
Banavar Sridhar ${ }^{*}$ \\ NASA Ames Research Center, Moffett Field, CA 94035-1000 \\ Hok K. $\mathrm{Ng}^{\dagger}$ \\ University of California, Santa Cruz, Moffett Field, CA 94035-1000 \\ and \\ Neil Y. Chen ${ }^{*}$ \\ NASA Ames Research Center, Moffett Field, CA 94035-1000
}

\begin{abstract}
There are indications that persistent contrails can lead to adverse climate change, although the complete effect on climate forcing is still uncertain. A flight trajectory optimization algorithm with fuel and contrails models, which develops alternative flight paths, provides policy makers the necessary data to make tradeoffs between persistent contrails mitigation and aircraft fuel consumption. This study develops an algorithm that calculates wind-optimal trajectories for cruising aircraft while avoiding the regions of airspace prone to persistent contrails formation. The optimal trajectories are developed by solving a non-linear optimal control problem with path constraints. The regions of airspace favorable to persistent contrails formation are modeled as penalty areas that aircraft should avoid and are adjustable. The tradeoff between persistent contrails formation and additional fuel consumption is investigated, with and without altitude optimization, for 12 city-pairs in the continental United States. Without altitude optimization, the reduction in contrail travel times is gradual with increase in total fuel consumption. When altitude is optimized, a two percent increase in total fuel consumption can reduce the total travel times through contrail regions by more than six times. Allowing further increase in fuel consumption does not seem to result in proportionate decrease in contrail travel times.
\end{abstract}

\section{Introduction}

$\mathrm{I}^{\mathrm{n}}$ nterest in the effect of aircraft condensation trails or contrails on climate change has increased in recent years. Aircraft emissions contain water vapor, carbon dioxide and other greenhouse gases. Contrails form in the wake of aircraft for various reasons but the most important is the emission of water vapor. ${ }^{1}$ They appear in the atmosphere along the aircraft's trajectory at high altitude where the ambient temperature is very low. Contrails persist in the region of atmosphere where the relative humidity with respect to ice is greater than $100 \%{ }^{2}$ A recent study ${ }^{3}$ reports that persistent contrails may have a three to four times greater effect on the climate than carbon dioxide emissions. It is tempting to reroute aircraft to minimize the impact of persistent contrails on climate. This may result in longer travel times, more fuel usage and increased carbon dioxide emissions. However, the complete effect of persistent contrails on climate change is still not known as they have both negative and positive effects and the resulting net effect is uncertain. The uncertainty in the effect of persistent contrails on climate forcing requires a flight trajectory optimization algorithm with fuel and contrails models that can develop alternative flight paths to enable tradeoff between persistent contrails mitigation and fuel consumption for policy makers to make acceptable aviation operation decisions.

Several new operational strategies in air traffic management have been proposed that can potentially mitigate the

\footnotetext{
*Senior Scientist for Air Transportation Systems, Aviation Systems Division, Fellow.

${ }^{\dagger}$ Senior Software Engineer, U.C. Santa Cruz, MS 210-8, Member AIAA.

${ }^{*}$ Aerospace Research Engineer, Systems Modeling and Optimization Branch, MS 210-10, Member AIAA.

1

American Institute of Aeronautics and Astronautics
} 
impact of persistent contrails on climate change. These strategies include adjusting cruise altitude in real-time ${ }^{4}$ and rerouting aircraft around regions of airspace that facilitate persistent contrails formation. ${ }^{5}$ The study in Ref. 6 presents a methodology to optimally reroute aircraft trajectories to avoid the formation of persistent contrails with the use of mixed integer programming. However, the computational complexity is very high for problems with many obstacles and dynamic constraints. None of the current methods for avoiding contrail $\mathrm{s}^{6-7}$ consider the effect of wind on the aircraft trajectory and neglect the potential fuel savings that aircraft can gain when flying wind-optimal routes.

This study develops an algorithm to calculate a wind-optimal trajectory for cruising aircraft while avoiding the regions of airspace that facilitate persistent contrails formation since expert panels ${ }^{3}$ have suggested focusing on the impacts of subsonic aviation emissions at cruise altitudes in the upper troposphere and lower stratosphere. The computationally efficient optimal trajectory is derived by solving a non-linear optimal control problem with path constraints. ${ }^{8}$ The regions of airspace favorable to persistent contrails formation are modeled as undesirable regions that aircraft should avoid and are formulated as soft state constraints. It is shown that the dynamical equation for aircraft optimal heading is reduced to the solution of the Zermelo problem ${ }^{9}$ in the absence of constrained airspace regions.

Section II provides the model for diagnosing regions of airspace that are susceptible for persistent contrail formation. Section III explains the optimal trajectory generation for cruising aircraft. Section IV describes the application of the trajectory optimization algorithm for calculating wind-optimal and contrails-avoidance routes. Conclusions and future work are described in Section V.

\section{Persistent Contrails Formation Models}

The formation of contrails has been under investigation since 1919. ${ }^{1}$ According to Appleman ${ }^{10}$, contrails are clouds that form when a mixture of warm engine exhaust gases and cold ambient air reaches saturation with respect to water, forming liquid drops, which quickly freeze. Contrails can persist when the ambient air is supersaturated with respect to ice i.e. the environmental relative humidity with respect to ice (RHi) is greater than $100 \%{ }^{2}$ In this study, the regions of airspace that have RHi greater than $100 \%$ are considered favorable to persistent contrails formation. The studies in Refs. 11-12 measure the validity of contrails formation by comparing them with satellite observation. There is general agreement between the satellite images and the persistent contrails regions predicted by the model.

The RHi is computed using measurements from the Rapid Update Cycle (RUC). RUC is an operational weather prediction system developed by the National Oceanic \& Atmospheric Administration (NOAA) for users needing frequently updated short-range weather forecasts (e.g. the US aviation community). The horizontal resolution in RUC is $40-\mathrm{km}$. RUC data has 37 vertical isobaric pressure levels ranging between 100-1000 mb in 25 $\mathrm{mb}$ increments. The RUC produces short-range forecasts every hour. RUC does not provide measurements for RHi directly. Instead, it has measurements for Relative Humidity with respect to water (RHw) and environmental temperatures. These measurements are used to compute the RHi by the following formula,

$$
R H i=R H w \cdot \frac{6.0612 e^{18.102 \cdot T /(249.52+T)}}{6.1162 e^{22.577 \cdot T /(273.78+T)}}
$$

where $T$ is temperature measured in Celsius. Note that the numerator on the right hand side of Eq. (1) is the saturation vapor pressure over water from the model denoted as $\operatorname{AERW}(50,-80)$ in Ref. 13 and the denominator is the saturation vapor pressure over ice from the model denoted as AERWi $(0,-80)$ in Ref. 13.

\section{Optimal Trajectory on Horizontal Plane}

Aircraft trajectory optimization algorithms are well-known and are solutions to two-point boundary value problems. ${ }^{9}$ The various approximations to the solution of the two-point boundary value problems depend on the application, and are motivated by the desire to balance computation speed with accuracy. The cruise altitude of most commercial aircraft varies between 29,000 feet to 41,000 feet. The flight levels are separated by 2000 feet between two levels of flight in the same direction (1000 feet since the introduction of Reduced Vertical Separation Minima). As the choice of the cruise altitude varies over a small range, the optimal aircraft trajectories in this paper are computed by repeatedly solving the horizontal plane problem.

This section develops the optimal trajectory algorithm for cruising aircraft. Section III.A presents the aircraft model and outlines the procedures for calculating optimal aircraft heading in a 2-dimensional plane. Section III.B 
models persistent contrails formation as regions to be avoided by an aircraft and imposes a soft penalty for going through these regions.

\section{A. Aircraft Model and Horizontal Trajectory Generation}

The equations of motion in the horizontal plane are

$$
\begin{gathered}
\dot{x}=V \cos \theta+u(x, y) \\
\dot{y}=V \sin \theta+v(x, y) \\
\dot{m}=-f
\end{gathered}
$$

subject to the conditions that $T h=\mathrm{D}$ and $\gamma=0$, where $x$ and $y$ are aircraft position in rectangular coordinates, $V$ is airspeed, $\theta$ is heading angle, $m$ is aircraft mass, $f$ is fuel flow rate, Th is thrust and $D$ is drag. The $\mathrm{x}$-component of the wind velocity is $u(x, y)$, and the y-component of the wind velocity is $v(x, y)$.

The horizontal trajectory is optimized by determining the heading angle that minimizes a cost function and satisfies the physical system constraints. The cost function contains components that penalize travelling time, fuel burn, and flying through penalty areas. ${ }^{16}$ The cost function is defined as

$$
J=\frac{1}{2} X^{T}\left(t_{f}\right) S X\left(t_{f}\right)+\int_{t_{0}}^{t_{f}}\left\{C_{t}+C_{f} f+C_{r} r(x, y)\right\} d t,
$$

where $X=\left[\begin{array}{ll}x & y\end{array}\right]^{T}$ is the state vector, $S$ is the final state cost matrix, $C_{t}$ is the cost coefficient of time, $C_{f}$ is the cost coefficient of fuel, $f$ is fuel flow rate, $C_{r}$ is the cost coefficient of penalty areas, and $r(x, y)$ is the penalty function. Section III.D discusses the penalty function in more detail. Using the chain rule of differentiation on the final state penalty cost, the cost function is re-written as

$$
J=\int_{t_{0}}^{t_{f}}\left[(S X(t))^{T} \dot{X}(t)+C_{t}+C_{f} f+C_{r} r(x, y)\right] d t .
$$

Pontryagin's Minimum Principle ${ }^{9}$ is applied to determine the control input that minimizes the cost function. The heading angle, $\theta$, is the control available for aircraft during cruise. The Hamiltonian for this problem is defined as

$$
H=(S X(t))^{T} \dot{X}(t)+C_{t}+C_{f} f+C_{r} r(x, y)+\lambda_{x}(V \cos \theta+u(x, y))+\lambda_{y}(V \sin \theta+v(x, y))+\lambda_{m}(-f),
$$

where $\lambda_{x}, \lambda_{y}$, and $\lambda_{\mathrm{m}}$ are the co-state parameters. The study in Ref.17 determined the value of $\lambda_{m}$ to be negligible during cruise portion of flight for transport-class aircraft. If $S$ is a diagonal matrix and $s_{x}$ and $s_{y}$ are the diagonal elements associated to the final position in $x$ and $y$ coordinates, the Hamiltonian for the reduced-order model is formulated as

$$
\begin{aligned}
H & =s_{x} x \dot{x}+s_{y} y \dot{y}+C_{t}+C_{f} f+C_{r} r(x, y)+\lambda_{x}(V \cos \theta+u(x, y))+\lambda_{y}(V \sin \theta+v(x, y)) \\
& =C_{t}+C_{f} f+C_{r} r(x, y)+\left(\lambda_{x}+s_{x} x\right)(V \cos \theta+u(x, y))+\left(\lambda_{y}+s_{y} y\right)(V \sin \theta+v(x, y)) .
\end{aligned}
$$

For an extremum to exist, the optimal heading angle satisfies

$$
\begin{aligned}
& \frac{\partial H}{\partial \theta}=0, \quad \mathrm{t}_{0} \leq t \leq t_{f} \\
\Rightarrow & \left(\lambda_{x}+s_{x} x\right) \sin \theta=\left(\lambda_{y}+s_{y} y\right) \cos \theta \\
\Rightarrow & \tan \theta=\frac{\left(\lambda_{y}+s_{y} y\right)}{\left(\lambda_{x}+s_{x} x\right)},
\end{aligned}
$$


and the necessary conditions for optimality are

$$
\begin{array}{ll}
H^{*}=\min \{H\}, & \text { in general } \\
H^{*}=0, & \text { free terminal time, } \mathrm{t}_{\mathrm{f}} .
\end{array}
$$

Solve Eq. (8-9) for the co-state parameters $\lambda_{x}$ and $\lambda_{y}$ when the Hamiltonian is zero to obtain

$$
\begin{aligned}
& \lambda_{x}=\frac{-\left(C_{t}+C_{f} f+C_{r} r(x, y)\right) \cos \theta}{V+u(x, y) \cos \theta+v(x, y) \sin \theta}-s_{x} x \\
& \lambda_{y}=\frac{-\left(C_{t}+C_{f} f+C_{r} r(x, y)\right) \sin \theta}{V+u(x, y) \cos \theta+v(x, y) \sin \theta}-s_{y} y .
\end{aligned}
$$

The co-state equations are

$$
\begin{aligned}
& \dot{\lambda}_{\mathrm{x}}=-\frac{\partial H}{\partial x} \Rightarrow \\
& -\dot{\lambda}_{x}=C_{r} \frac{\partial r(x, y)}{\partial x}+\left(\lambda_{x}+s_{x} x\right)\left(\frac{\partial u(x, y)}{\partial x}\right)+s_{x}(V \cos \theta+u(x, y))+\left(\lambda_{y}+s_{y} y\right)\left(\frac{\partial v(x, y)}{\partial x}\right) \\
& \dot{\lambda}_{y}=-\frac{\partial H}{\partial y} \Rightarrow \\
& -\dot{\lambda}_{y}=C_{r} \frac{\partial r(x, y)}{\partial y}+\left(\lambda_{x}+s_{x} x\right)\left(\frac{\partial u(x, y)}{\partial y}\right)+\left(\lambda_{y}+s_{y} y\right)\left(\frac{\partial v(x, y)}{\partial y}\right)+s_{y}(V \sin \theta+v(x, y))
\end{aligned}
$$

Equations $(9,13,14)$ are known as the Euler-Lagrange equations. As shown in the Appendix, the dynamical equation for the optimal aircraft heading is governed by Eqs. (9-15), and the heading angle, $\theta$, is the solution to the differential equation

$$
\begin{aligned}
\dot{\theta}=\frac{(V+u(x, y) \cos \theta+v(x, y) \sin \theta)}{\left(C_{t}+C_{f} f+C_{r} r(x, y)\right)} & \left(-C_{r} \sin \theta \frac{\partial r(x, y)}{\partial x}+C_{r} \cos \theta \frac{\partial r(x, y)}{\partial y}\right) \\
& +\sin ^{2} \theta\left(\frac{\partial v(x, y)}{\partial x}\right)+\sin \theta \cos \theta\left(\frac{\partial u(x, y)}{\partial x}-\frac{\partial v(x, y)}{\partial y}\right)-\cos ^{2} \theta\left(\frac{\partial u(x, y)}{\partial y}\right) .
\end{aligned}
$$

Note that this equation is reduced to that in the Zermelo problem ${ }^{9}$ when $C_{r}=0$. Solve Eqs. $(2,3,15)$ simultaneously to obtain the optimal path. The initial heading angle at $t_{0}$ must be picked correctly for a particular origin and destination pair. This study applies the shooting method in Ref. 18 to find the initial aircraft heading.

\section{B. Contrails as Penalty Areas}

Persistent contrails are modeled using penalty functions as areas to be avoided by an aircraft to reduce the potential impact on climate. The cost due to persistent contrails formation in the cost function is defined as

$$
J_{r}=\int_{t_{0}}^{t_{f}} C_{r} r(x, y) d t
$$

The penalty function $r(x, y)$ is the penalties that an aircraft can encounter along the flight trajectory from the origin to destination. In general, there are multiple regions in the en-route airspace that favor persistent contrails formation. The penalty can be determined by one of the following functions: 
1. Radial penalty functions-

$$
\begin{gathered}
r(x, y)=\sum_{i} \frac{1}{d_{i}}, \text { or } \\
r(x, y)=\sum_{i} \frac{1}{d_{i}^{2}},
\end{gathered}
$$

where $d_{i}$ is distance between the aircraft and the center of $i^{\text {th }}$ region that potentially form persistent contrails. 2. Uniform penalty function-

$$
r(x, y)=\left\{\begin{array}{cc}
\text { Constant } & \text { Aircraft in penalty area } \\
0 & \text { Otherwise }
\end{array}\right.
$$

In this study, optimal trajectories will be generated using the penalty function defined in Eq. (17). In addition, there are many regions in the NAS that can potentially form persistent contrails. Some are far away from the aircraft and will not be encountered by the aircraft. Some are expected to be encountered, but the area is too large for aircraft to completely avoid. Identifying the right subset of contrails and avoiding the region by an appropriate level are important for policy makers to make operational decisions and tradeoffs.

\section{Aircraft Fuel Consumption Model}

This study applies the fuel consumption model in Eurocontrol's Base of Aircraft Data Revision 3.6 (BADA) ${ }^{19}$ to compute cruising aircraft fuel consumption. The following equation calculates fuel burn for aircraft during cruise

$$
f_{c}=t \cdot S F C \cdot T h
$$

where $f_{c}$ is the fuel burn during cruise, $t$ is elapsed time, Th is thrust, and $S F C$ is the specific fuel consumption. As an example, in Figure 1, the fuel burn (in $\mathrm{Kg}$ ) for a typical short to medium jet airliner from Chicago to New York, is shown as a function of altitude for three different weights, Low, Medium and High.

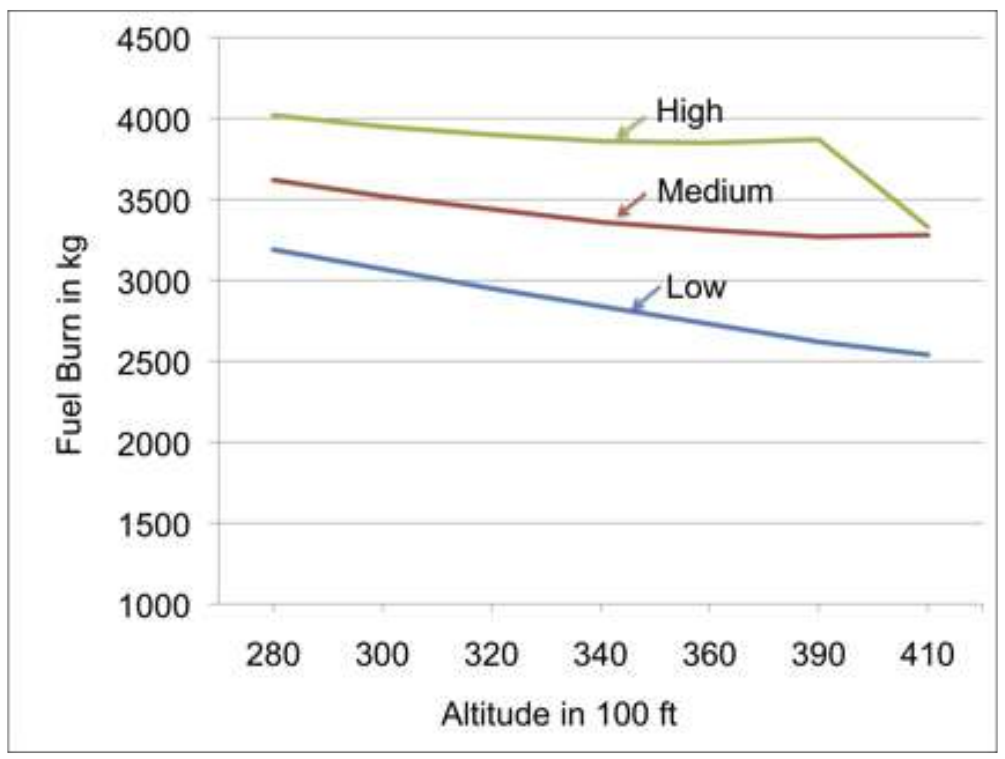

Figure 1. Fuel flow as a function of altitude 


\section{Results}

This section presents results based on applying the optimal trajectory algorithm to calculate an aircraft trajectory in the presence of winds that avoids regions of airspace that facilitate persistent contrails formation. The trajectory computations are done using traffic and atmospheric data in the continental United States for May 24, 2007. The data for wind speed and direction are obtained from RUC. The blue, green and magenta polygons in Fig. 2 depict the areas at 33,000 feet above sea level in the U.S. national airspace where RHi is greater than $100 \%$ at 6 a.m., 7 a.m. and 8 a.m. EDT on May 24, 2007, respectively. The RHi values are computed using Eq. (1) with RHw values and temperature data obtained from RUC. Figure 2 shows that the location, shape and size of potential contrail regions vary with time.

Section IV.A discusses contrail avoidance trajectories from Chicago to New York for 10 different flight altitudes. Section IV.B presents results on partial and complete contrail avoidance trajectories for a longer route from Los Angeles to New York. Section IV.C applies the methodology to a group of flights covering 12 city-pairs.

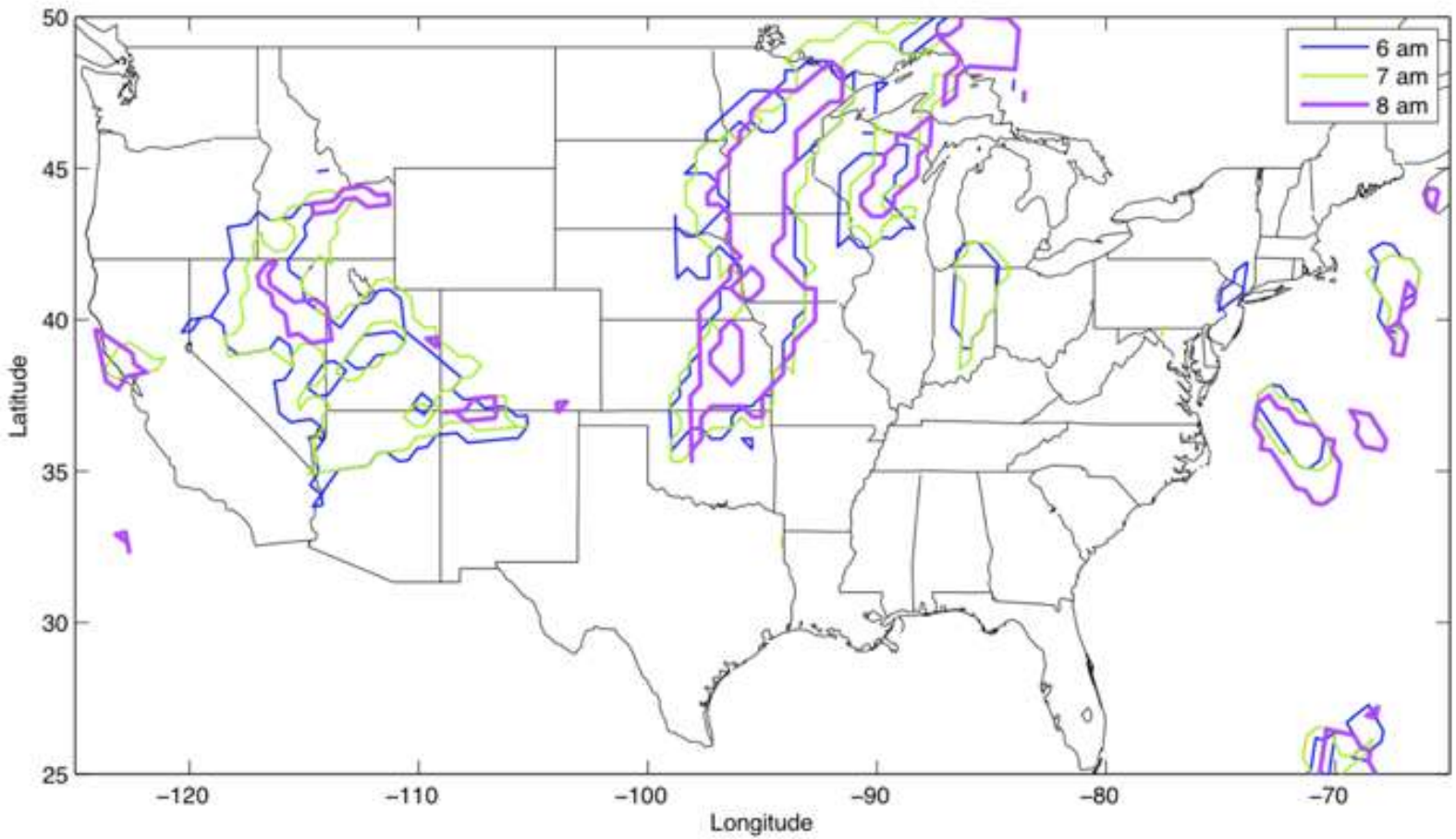

Figure 2. Regions of airspace at 33,000 feet where RHi is greater than $100 \%$ at 6 a.m., 7 a.m., and 8 a.m. on May 24, 2007.

\section{A. Chicago (O'Hare Airport) to New York (Newark Liberty Airport)}

The trajectory design in this example focuses on avoiding the potential contrails regions between Chicago and New York. At each altitude, $C_{r}$ is varied from zero to a value where the optimal trajectory completely avoids the contrail region. The time for the wind optimal route, the time through contrail regions, the value of $C_{r}$ to avoid contrails and the extra travelling time are computed at each altitude. This process is repeated at all the ten altitudes. Three optimal trajectories from Chicago O'Hare airport (ORD) to Newark Liberty airport (EWR) are shown in Fig. 3 for flights with cruising altitude equal to 34,000 feet. The cruising speed is assumed to be $400 \mathrm{nmi} / \mathrm{hr}(741 \mathrm{~km} / \mathrm{hr})$. The green arrows represent the wind directions, obtained from RUC, at 6 a.m. EDT on May 24, 2007. The arrow sizes are plotted in proportion to the wind magnitudes. The wind-optimal trajectory is generated using Eqs. (2, 3, $15)$ by setting $C_{r}=0$. Two optimal trajectories in additional to the wind-optimal route are also plotted in Fig. 3. The areas favorable to persistent contrails formation ( $\mathrm{RHi}>100 \%$ ) are surrounded by the blue polygons. The polygon with a red cross is identified as a potential penalty area to the aircraft and the red-cross is the center of penalty area. The position of penalty centers and aircraft position are used in Eq. (17) to calculate the distance and the penalty cost. In this example, the cost coefficient of time is chosen as $C_{t}=20$. The cost coefficient of penalty $C_{r}$ is equal to 0.2 and 0.6 , respectively. Note that the penalty coefficient $C_{r}$ is treated as a design parameter. The choice of this parameter is not unique and depends on the definition of the penalty function itself. The optimal route 
with $C_{r}=0.6$ completely avoids the contrail polygons near the departure airport. The optimal route with $C_{r}=0.2$ only partially avoids the polygons but is shorter. Note that both routes travel through the blue polygon surrounding the destination where aircraft start to land. In this case, there is a tradeoff between flying a shorter route with more persistent contrails formation versus flying a longer route with less persistent contrails formation. The performance of optimal trajectories is evaluated by investigating the total travel time and the time associated traveling through regions of persistent contrails formation.

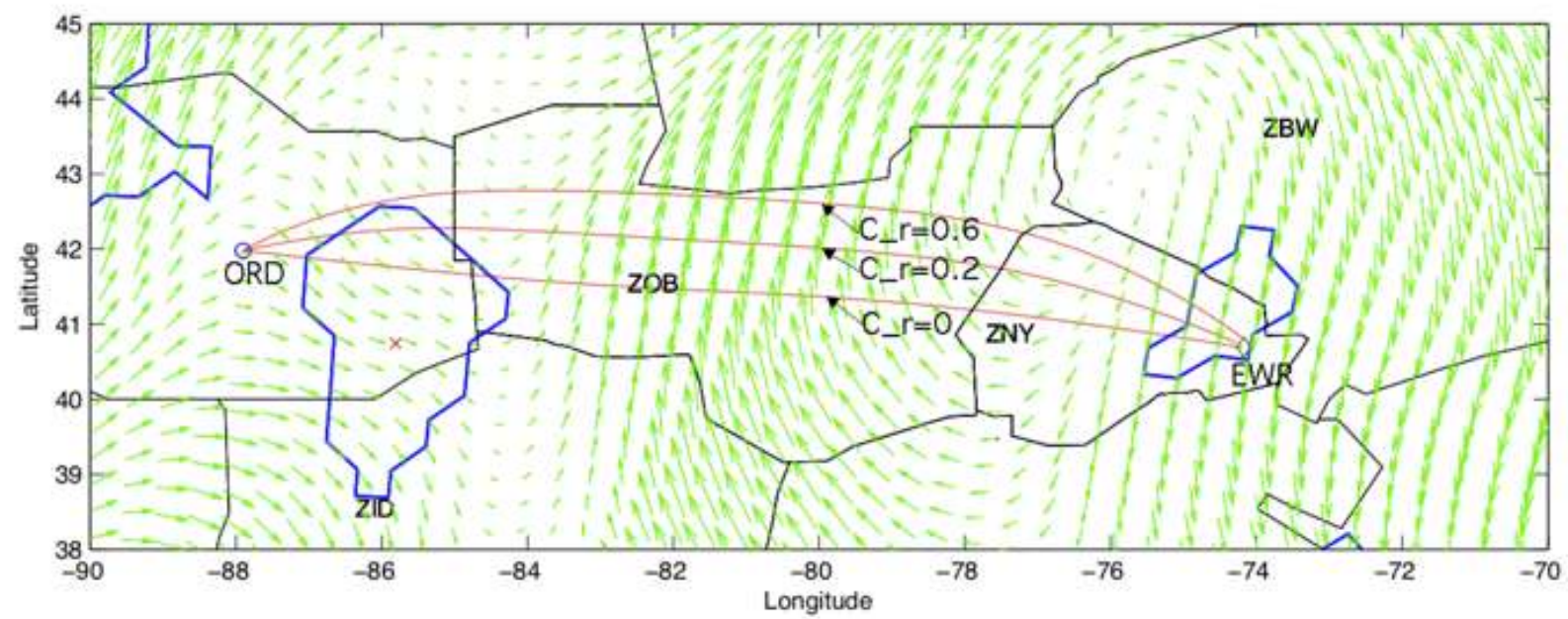

Figure 3. Optimal trajectories at 34,000 feet from ORD to EWR with different design parameters.

Optimal aircraft trajectories are generated for 10 different altitudes between 28,000 feet and 52,000 feet (i.e. corresponds to the isobaric pressure level between $325 \mathrm{hpa}$ and $100 \mathrm{hpa}$ with $25 \mathrm{hpa}$ increments). Figure 4 shows the results for the 10 wind-optimal trajectories. The sum of blue and red bars represents the total travel time for each trajectory, and the red bar presents the length of periods that a flight travels inside the regions of airspace favorable to persistent contrails formation. The wind-optimal trajectories at $28,000 \mathrm{ft}, 41,000 \mathrm{ft}, 44,000 \mathrm{ft}, 48,000 \mathrm{ft}$ and 52,000 ft do not intercept any region of airspace that facilitates persistent contrails formation. The flights at these cruising altitudes should fly the wind-optimal trajectories that minimize fuel burn and emissions. Flying wind optimal trajectories at other altitudes between $30,000 \mathrm{ft}$ and 39,000 ft will potentially cause persistent contrails formation. Optimal contrails-avoidance trajectories at these altitudes are generated by increasing the value of $C_{r}$ from 0 to 2 with increments equal to 0.2 .

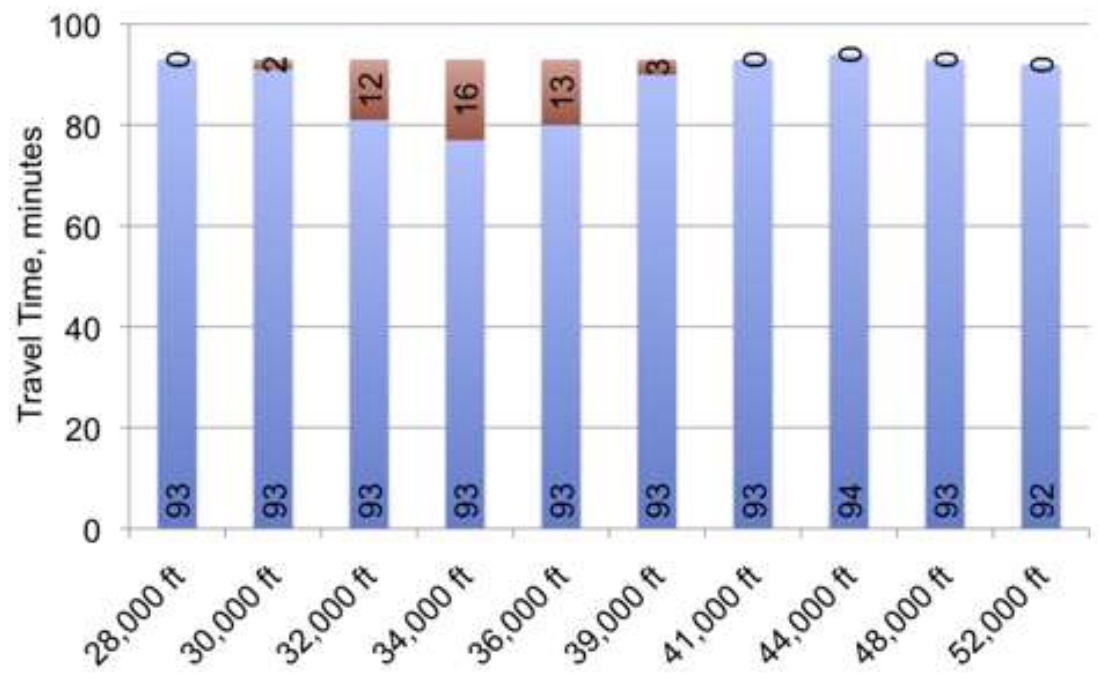

Figure 4. Travel time for the wind-optimal routes from ORD to EWR and length of periods favorable to persistent contrails formation at 10 different altitudes. 
The green columns in Table 1 shows the value of $C_{r}$, the total travel time for the contrails-avoidance trajectory and the additional travel time compared to that of wind-optimal trajectory. The blue columns show the travel time for wind-optimal trajectories. The additional travel times range from 0 to $4.3 \%$ with a corresponding increase in fuel from 0 to $4.46 \%$. Flying a contrails-avoidance trajectory requires very small additional travel time at 39,000 feet to avoid potentially 3 minutes of persistent contrails formation. Flying a contrails-avoidance trajectory at 34,100 feet requires $4.3 \%$ more travel time to avoid formation of 16 minutes of persistent contrails. More optimal trajectories can be calculated with various choice of $C_{r}$.

Table 1. Wind-optimal trajectories (in blue) versus contrails-avoidance trajectories (in green) from ORD to EWR.

\begin{tabular}{|l|c|c|c|c|c|c|c|c|c|c|}
\hline \hline Altitude (ft) & \multicolumn{2}{|c|}{30,000} & \multicolumn{2}{|c|}{32,000} & \multicolumn{2}{c|}{34,000} & \multicolumn{3}{c|}{36,000} & \multicolumn{2}{c|}{39,000} \\
\hline$C_{r}$ & 0 & 0.4 & 0 & 1.6 & 0 & 0.6 & 0 & 1.8 & 0 & 0.2 \\
\hline Trajectory (minute) & 93 & 94 & 93 & 95 & 93 & 97 & 93 & 95 & 93 & 93 \\
\hline Contrails (minute) & 2 & 0 & 12 & 0 & 16 & 0 & 13 & 0 & 3 & 0 \\
\hline Additional Travelling Time (\%) & 0 & 1.1 & 0 & 2.2 & 0 & 4.3 & 0 & 2.2 & 0 & 0 \\
\hline Fuel Burn (Kg) & 3520 & 3560 & 3440 & 3510 & 3360 & 3510 & 3310 & 3380 & 3270 & 3271 \\
\hline Additional Fuel Burn (\%) & 0 & 1.13 & 0 & 2.03 & 0 & 4.46 & 0 & 2.11 & 0 & 0 \\
\hline
\end{tabular}

\section{B. Los Angeles (International Airport) to New York (Newark Liberty Airport)}

The flight from ORD to EWR illustrated a strategy on completely avoiding contrails. Avoiding the contrails partially results in lower extra fuel consumption over wind optimal routes. This section describes the trade-off between incurring extra fuel costs and partial avoiding of contrails. The optimal trajectory that minimizes potential climate change can then be determined when the relative severity of contrails and emissions on climate impact is known. Generating and comparing these optimal trajectories at different flight altitudes provides policy makers with the necessary data to make tradeoffs between persistent contrails mitigation and fuel consumption.

Partial to complete contrail avoidance versus extra fuel consumption analysis is presented for a flight departing from Los Angeles (LAX) to New York (EWR) at 4:00AM on May 24, 2007. Three optimal trajectories from LAX to EWR are shown in Fig. 5 for flights with cruising altitude equal to 35,000 feet. Optimal horizontal trajectories are generated at six different flight levels from $29,000 \mathrm{ft}$ to $39,000 \mathrm{ft}$ for varying values of $C_{r}$ from zero to 0.25 in increments of 0.05 . For each altitude and $C_{r}$ combination, the amount of extra fuel consumed over wind optimal routes, $\Delta \mathrm{f}$, and the corresponding amount of travel through potential regions of contrail formation, $\Delta \mathrm{c}$, are computed. Figure 6 shows contour plots of $\Delta \mathrm{c}$ and $\Delta \mathrm{f}$ as functions of altitude and $C_{r}$. The plots illustrate the non-linear dependence of $\Delta \mathrm{c}$ and $\Delta \mathrm{f}$ as a function of altitude and $C_{r}$.

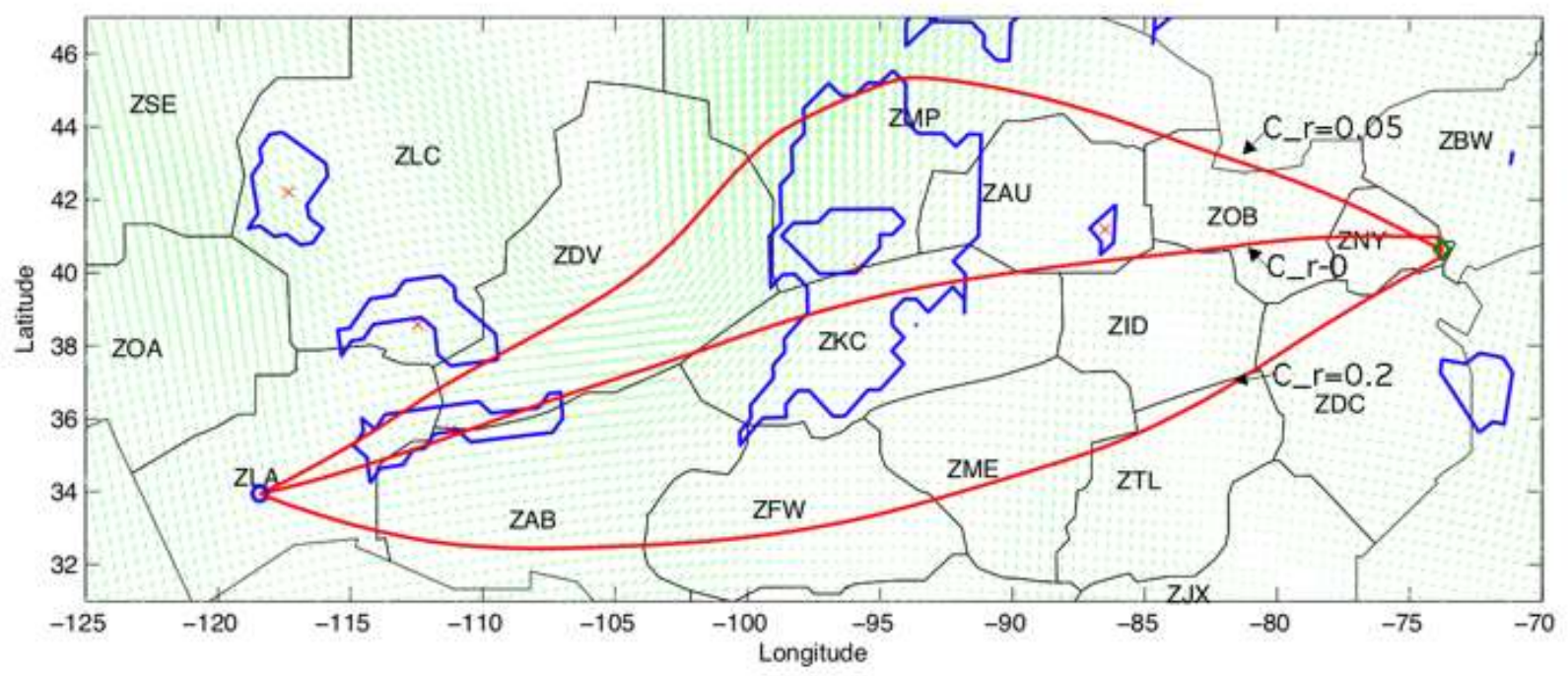

Figure 5. Optimal trajectories from Los Angeles to New York with different design parameters. 

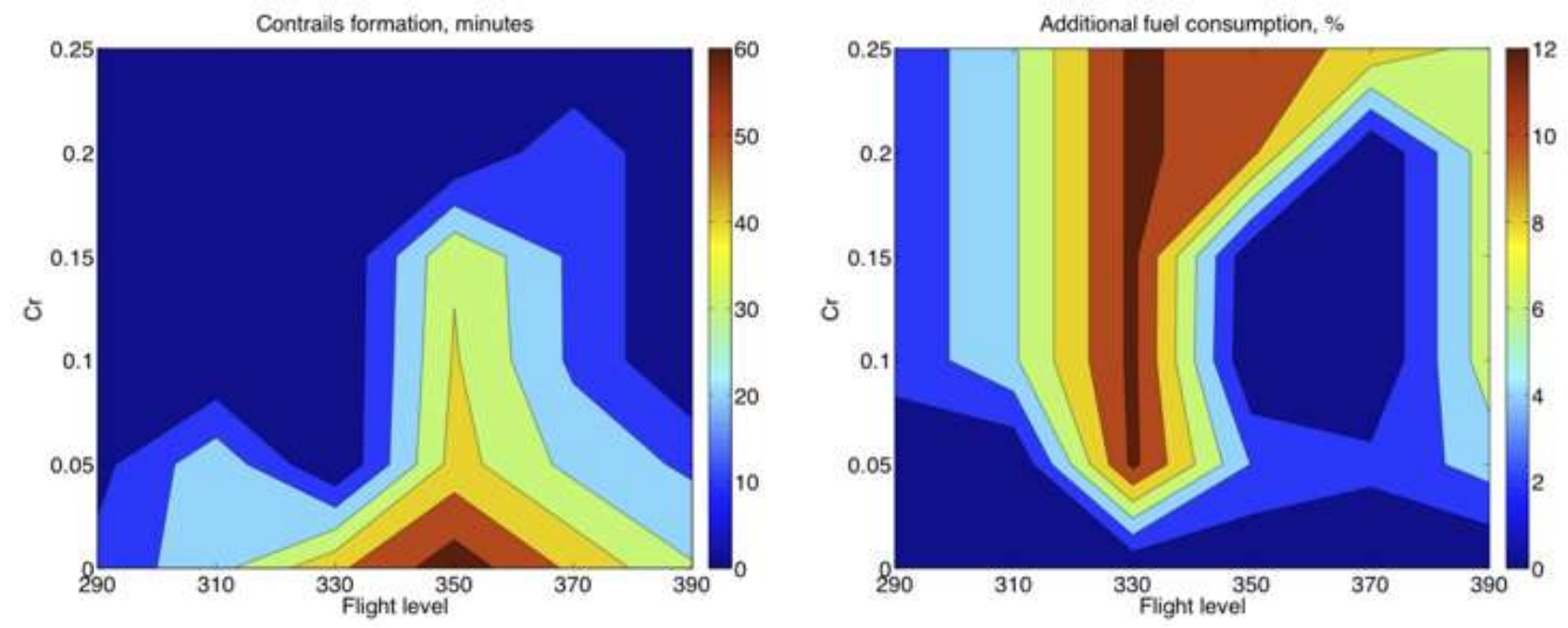

Figure 6. Contours of $\Delta \mathrm{c}$ and $\Delta \mathrm{f}$ as functions of altitude and $C_{r}$

The plots show the amount of contrails formed if there is a limit on the extra amount of fuel consumed. Zero additional fuel consumption corresponds to $C_{r}=0$, and results in zero to 60 minutes of contrail formation depending on the flight level. Increasing the limit on extra fuel consumption further reduces contrails formation. An extra 2 percent to 12 percent fuel burnt reduces contrails formation to zero for these altitudes. The fuel cost and environmental cost due to aircraft emissions are proportional to the total travel time, and the environmental cost of persistent contrails formation depends on the contrails formation time. Calculating the additional fuel consumption and the time associated with persistent contrails formation for a set of optimal trajectories with various $C_{r}$ provides critical data required for optimal trajectory selection. One can choose the right trajectory that minimizes the climate impact when the relative magnitude of climate impact by emissions and persistent contrails formation is known.

\section{Optimal Trajectories for 12 City Pairs}

This section analyzes the wind-optimal and contrails-avoidance trajectories for 12 origin-destination pairs for a period of 24 hours starting from 6 a.m. EDT on May 24, 2007. The same city-pairs were used by the Federal

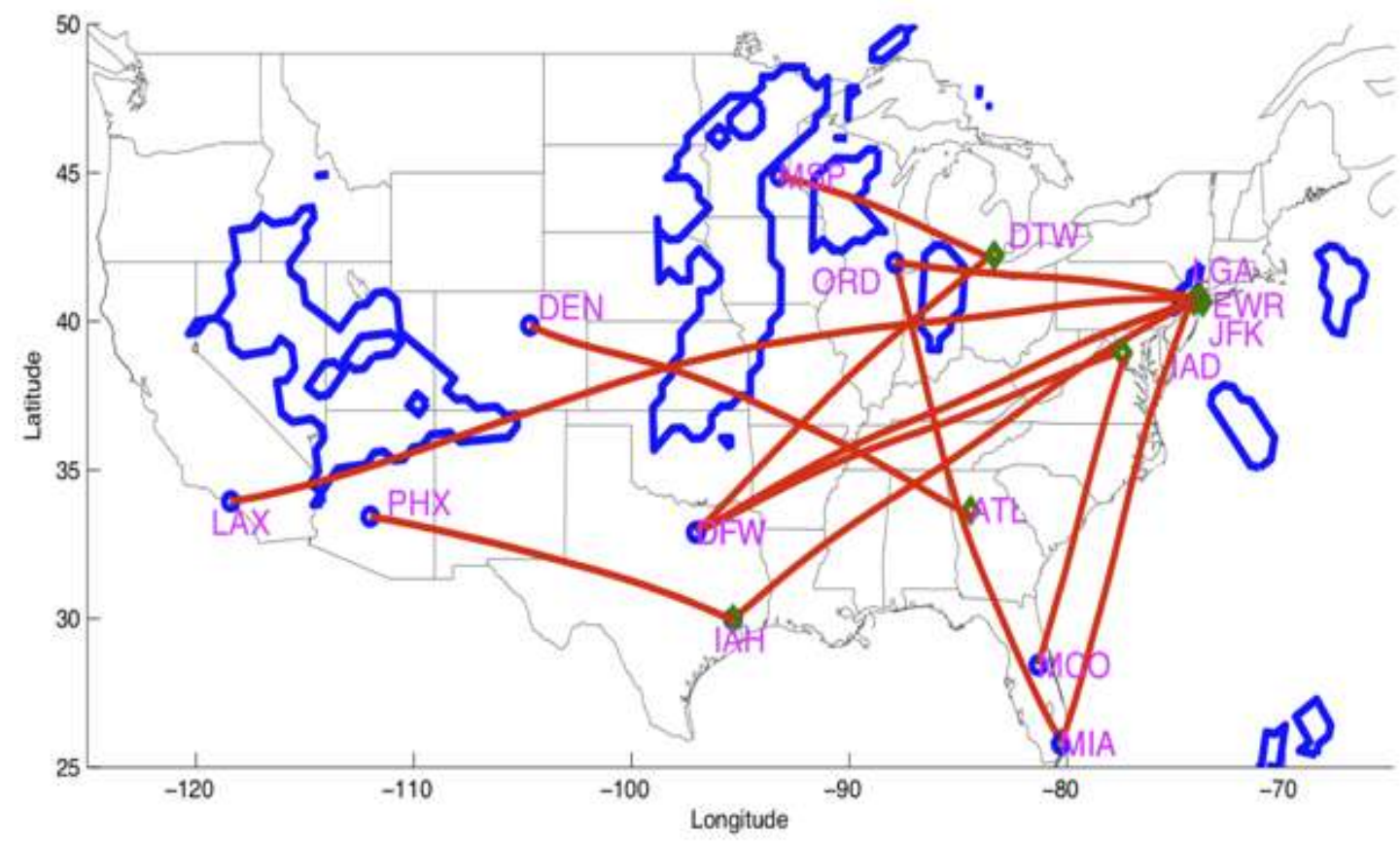

Figure 7. The wind-optimal trajectories for the eastbound flights for 12 city pairs on 33,000 feet at 6 a.m. EDT on May 24, 2007. 
Aviation Administration to assess the impact of implementation of Reduced Vertical Separation Minima (RVSM) on aircraft-related fuel burn and emissions. ${ }^{20}$ This part of the study adapts the standard in RVSM and assumes that the cruising altitudes are between 29,000 and 41,000 feet. Eastbound aircraft fly odd thousands of feet while westbound traffic fly even thousands of feet. Figure 7 shows the wind-optimal trajectories for the eastbound flights at 33,000 feet at 6 a.m. EDT. Blue polygons depict the areas favorable to persistent contrails formation (RHi > $100 \%)$.

The optimal aircraft trajectories are generated at the beginning of each hour on May 24, 2007 using hourly updated weather data from RUC. Six flight levels are considered for each direction of air traffic for each city pair. A group of 21 optimal aircraft trajectories are calculated for each flight level by increasing the value of $C_{r}$ from 0 to 2 with increments equal to 0.1 . The cost coefficient of time is chosen as $C_{t}=20$ for each case. The cruising speed is assumed to be $420 \mathrm{nmi} / \mathrm{hr}$. The fuel consumption for each aircraft trajectory is calculated using BADA formulas by assuming that the aircraft are short to medium range jet airliners with medium weight. In each group, the additional fuel consumption of each optimal trajectory is obtained by comparing its fuel burnt to that of its wind-optimal trajectory $\left(C_{r}=0\right)$. The persistent contrails formation time associated to each trajectory is also recorded.

Table 2. Average persistent contrails formation time (minutes) for the optimal aircraft trajectories for the 12 cities on May 24, 2007.

\begin{tabular}{|c|c|c|c|c|c|c|c|c|c|c|c|c|}
\hline & \multirow{2}{*}{ City-pair } & \multicolumn{11}{|c|}{ Maximum Additional Fuel Consumption (\%) } \\
\hline & & $\mathbf{0}$ & 2 & & 4 & & & & (2) & 3 & & \\
\hline \multirow{24}{*}{ 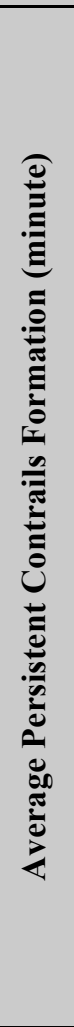 } & LAX to JFK & 18.3 & 13.4 & 2.2 & 10.6 & 1.1 & 8.6 & 0.9 & 6.0 & \begin{tabular}{|c|}
0.5 \\
\end{tabular} & 0.2 & 0.0 \\
\hline & JFK to LAX & 26.2 & 16.6 & 1.6 & 10.8 & 0.8 & 6.3 & 0.2 & 4.8 & 0.1 & 0.7 & $\mathbf{0 . 0}$ \\
\hline & ATL to DEN & 18.9 & 13.7 & 3.7 & 11.3 & 2.3 & 9.8 & 1.2 & 8.8 & 0.8 & 3.1 & $\overline{0.0}$ \\
\hline & DEN to ATL & 14.0 & 9.7 & 2.2 & 8.8 & 1.4 & 8.0 & $\mathbf{1 . 0}$ & 7.0 & 0.5 & 3.3 & $\mathbf{0 . 0}$ \\
\hline & PHX to IAH & 11.5 & 9.7 & 6.2 & 7.9 & 5.3 & 7.3 & 4.8 & 7.2 & \begin{tabular}{|l|}
4.5 \\
\end{tabular} & 5.3 & 2.2 \\
\hline & IAH to PHX & 10.6 & 8.8 & 3.7 & 7.3 & 3.1 & 6.7 & 2.6 & 6.5 & 2.2 & 4.0 & 0.9 \\
\hline & DTW to DFW & 5.6 & 3.2 & 0.7 & 2.3 & 0.3 & 1.7 & 0.2 & 1.3 & \begin{tabular}{|l|}
0.1 \\
\end{tabular} & 0.2 & 0.0 \\
\hline & DFW to DTW & 7.9 & 4.9 & 0.9 & 2.8 & 0.4 & 1.7 & 0.2 & 1.0 & 0.1 & 0.3 & $\overline{0.0}$ \\
\hline & DFW to LGA & 1.9 & 1.2 & $\mathbf{0 . 0}$ & 0.7 & 0.0 & 0.6 & 0.0 & 0.6 & 0.0 & 0.3 & 0.0 \\
\hline & LGA to DFW & 2.5 & 1.5 & 0.0 & 0.9 & 0.0 & 0.5 & 0.0 & 0.3 & 0.0 & 0.0 & $\overline{0.0}$ \\
\hline & ORD to LGA & 1.7 & 0.9 & 0.1 & 0.6 & 0.1 & 0.4 & 0.0 & 0.4 & 0.0 & 0.2 & 0.0 \\
\hline & LGA to ORD & 2.2 & 1.0 & 0.5 & 0.7 & 0.3 & 0.6 & 0.2 & 0.4 & 0.2 & 0.4 & 0.0 \\
\hline & MSP to DTW & 1.7 & 1.4 & 0.0 & 1.2 & 0.0 & 1.0 & 0.0 & 0.9 & 0.0 & 0.2 & 0.0 \\
\hline & DTW to MSP & 1.8 & 1.5 & 0.0 & 1.3 & 0.0 & 1.0 & 0.0 & 1.0 & 0.0 & 0.6 & 0.0 \\
\hline & DFW to IAD & 1.5 & 0.9 & $\mathbf{0 . 0}$ & 0.7 & 0.0 & 0.4 & 0.0 & 0.4 & 0.0 & 0.2 & $\overline{0.0}$ \\
\hline & IAD to DFW & 1.9 & 1.1 & 0.0 & 0.7 & 0.0 & 0.3 & 0.0 & 0.2 & 0.0 & 0.0 & 0.0 \\
\hline & IAH to EWR & 1.9 & 1.5 & 0.0 & 1.3 & 0.0 & 1.2 & 0.0 & 1.2 & 0.0 & 0.8 & 0.0 \\
\hline & EWR to IAH & 0.6 & 0.4 & $\mathbf{0 . 0}$ & 0.2 & 0.0 & 0.1 & 0.0 & 0.1 & 0.0 & 0.1 & 0.0 \\
\hline & ORD to MIA & 0.4 & 0.1 & 0.0 & 0.0 & 0.0 & 0.0 & 0.0 & 0.0 & 0.0 & 0.0 & $\mathbf{0 . 0}$ \\
\hline & MIA to ORD & 0.5 & 0.2 & 0.0 & 0.0 & 0.0 & 0.0 & 0.0 & 0.0 & $\begin{array}{ll}0.0 \\
\end{array}$ & 0.0 & 0.0 \\
\hline & MIA to EWR & 0.1 & 0.0 & $\mathbf{0 . 0}$ & 0.0 & 0.0 & 0.0 & 0.0 & 0.0 & 0.0 & 0.0 & $\mathbf{0 . 0}$ \\
\hline & EWR to MIA & 0.1 & 0.0 & 0.0 & 0.0 & 0.0 & 0.0 & 0.0 & 0.0 & 0.0 & 0.0 & 0.0 \\
\hline & IAD to MCO & 0.0 & 0.0 & 0.0 & 0.0 & 0.0 & 0.0 & 0.0 & 0.0 & 0.0 & 0.0 & 0.0 \\
\hline & MCO to IAD & 0.0 & 0.0 & $\mathbf{0 . 0}$ & 0.0 & 0.0 & 0.0 & 0.0 & 0.0 & 0.0 & $\mathbf{0 . 0}$ & 0.0 \\
\hline
\end{tabular}

A total of six bins are defined such that the aircraft trajectories can be categorized based on their additional fuel consumption. The first bin contains the wind-optimal trajectory, which is the baseline for fuel use comparison and corresponds to trajectories that require zero \% of additional fuel consumption. The second bin contains aircraft trajectories that consume less than $2 \%$ additional fuel, the third bin contains those consume less than $4 \%$, and etc. The sixth bin has trajectories that burn more than $8 \%$ of fuel. In each bin, the optimal trajectory that has least amount of persistent contrails formation time is selected to represent the bin. Note that there are six bins for each 
group of trajectories and six groups for each direction of air traffic every hour. The average persistent contrails formation time for the optimal trajectories are summarized in Table 2. The white column presents the average contrails formation time measured in minutes for the wind-optimal trajectories. The average is taken over 6 flight levels and a period of 24 hours for each direction. Flying wind-optimal routes on any of the 6 flight levels from IAD to $\mathrm{MCO}$ or in the opposite direction do not induce persistent contrails formation that day. The contrails formation time is less than a minute on average for the city pairs such as ORD-MIA and MIA-EWR. The blue column presents the average contrails formation time for the optimal contrails-avoidance trajectories. These optimal trajectories consume different amounts of additional fuel at each bin or column. In general, the flights, which can afford more additional fuel burn, induce fewer contrails since they have more routes to choose from. The green column presents the average contrails formation time for the optimal contrails-avoidance trajectories on the optimized altitudes that produce least amount of contrails. The flights, which can select the flying altitudes, induce much fewer contrails given the same amount of extra fuel.

The data in Table 2 can be converted into actual amount of extra fuel consumed by various flights and then can be aggregated to produce a simplified total extra fuel consumption versus total minutes through the contrail regions for all the flights between the 12 city-pairs. Figure 8 shows the curves with (green curve) and without (blue curve) altitude optimization.

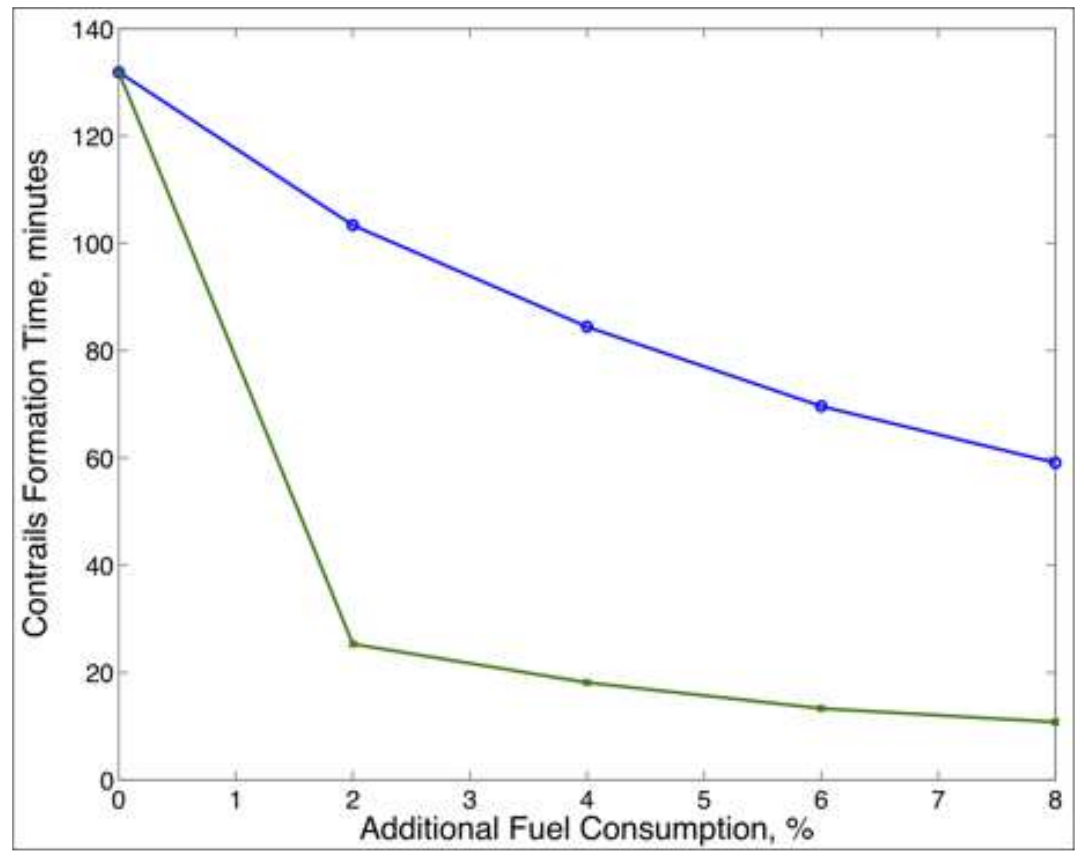

Figure 8. Trade-off curves between fuel consumption and contrail avoidance.

The figure shows, when altitude is optimized, a two percent increase in total fuel consumption can reduce the total travel times through contrail regions from 130 minutes to 22 minutes. Allowing further increase in fuel consumption does not seem to result in proportionate decrease in contrail travel times. Without altitude optimization, the reduction in contrail travel times is gradual with increase in total fuel consumption. The data in Table 2 can be used to develop optimal fleet allocation and scheduling strategies to minimize the travel time through contrails.

\section{Conclusion}

This study develops an algorithm to calculate wind-optimal trajectories for aircraft while avoiding the regions of airspace that facilitate persistent contrails formation. The optimal trajectory is derived by solving a non-linear optimal control problem with path constraints. The operational strategies investigated in this study for minimizing aviation impacts on climate change include flying wind-optimal routes, avoiding complete or partial persistent contrails formation and altering cruising altitudes. The tradeoff between persistent contrails formation and additional travel time are investigated at 10 different cruising altitudes for flights from Chicago to New York. The additional

11

American Institute of Aeronautics and Astronautics 
travel times required for completely avoiding persistent contrails formation at various flight altitudes ranged from approximately $0 \%$ to $4.3 \%$ for flights from Chicago to New York. The tradeoff between persistent contrails formation and additional fuel consumption is investigated, with and without altitude optimization, for 12 city-pairs in the continental United States. When altitude is optimized, a two percent increase in total fuel consumption can reduce the total travel times through contrail regions by more than six times. Allowing a further increase in fuel consumption does not seem to result in a proportionate decrease in contrail travel times. Without altitude optimization, the reduction in contrail travel times is gradual with an increase in total fuel consumption.

The results in this paper were based on traffic for a single day and used the same type of aircraft on all routes. The results can be modified using the complete traffic and weather data for extended periods of time to get a better understanding of the complex relation between fuel efficiency and the impact on the environment.

\section{Appendix}

The dynamical equation for the optimal aircraft heading is obtained using Eqs. (9-14). Differentiate both sides of Eq. (9) gives

$$
\begin{gathered}
\sec ^{2} \theta \dot{\theta}=\frac{\left(\dot{\lambda}_{y}+s_{y} \dot{y}\right)\left(\lambda_{x}+s_{x} x\right)-\left(\lambda_{y}+s_{y} y\right)\left(\dot{\lambda}_{x}+s_{x} \dot{x}\right)}{\left(\lambda_{x}+s_{x} x\right)^{2}} \\
\Rightarrow \dot{\theta}=\frac{\cos ^{2} \theta\left[\left(\dot{\lambda}_{y}+s_{y} \dot{y}\right)\left(\lambda_{x}+s_{x} x\right)-\left(\lambda_{y}+s_{y} y\right)\left(\dot{\lambda}_{x}+s_{x} \dot{x}\right)\right]}{\left(\lambda_{x}+s_{x} x\right)^{2}} .
\end{gathered}
$$

Substitute

$$
\lambda_{x}+s_{x} x=\frac{-\left(C_{t}+C_{f} f+C_{r} r(x, y)\right) \cos \theta}{V+u(x, y) \cos \theta+v(x, y) \sin \theta} \text { and } \lambda_{y}+s_{y} y=\frac{-\left(C_{t}+C_{f} f+C_{r} r(x, y)\right) \sin \theta}{V+u(x, y) \cos \theta+v(x, y) \sin \theta}
$$

from Eqs. (11-12) into Eq. (A1) to get

$$
\dot{\theta}=\frac{(V+u(x, y) \cos \theta+v(x, y) \sin \theta)\left[-\left(\dot{\lambda}_{y}+s_{y} \dot{y}\right) \cos \theta+\left(\dot{\lambda}_{x}+s_{x} \dot{x}\right) \sin \theta\right]}{\left(C_{t}+C_{f} f+C_{r} r(x, y)\right)} .
$$

We have from Eqs. (13-14),

$$
\begin{aligned}
\dot{\lambda}_{x}+s_{x} \dot{x} & =-C_{r} \frac{\partial r(x, y)}{\partial x}-\left(\lambda_{x}+s_{x} x\right)\left(\frac{\partial u(x, y)}{\partial x}\right)-\left(\lambda_{y}+s_{y} y\right)\left(\frac{\partial v(x, y)}{\partial x}\right) \\
& =-C_{r} \frac{\partial r(x, y)}{\partial x}+\frac{\left(C_{t}+C_{f} f+C_{r} r(x, y)\right) \cos \theta}{V+u(x, y) \cos \theta+v(x, y) \sin \theta}\left(\frac{\partial u(x, y)}{\partial x}\right)+\frac{\left(C_{t}+C_{f} f+C_{r} r(x, y)\right) \sin \theta}{V+u(x, y) \cos \theta+v(x, y) \sin \theta}\left(\frac{\partial v(x, y)}{\partial x}\right), \text { and } \\
\dot{\lambda}_{y}+s_{y} \dot{y} & =-C_{r} \frac{\partial r(x, y)}{\partial y}-\left(\lambda_{x}+s_{x} x\right)\left(\frac{\partial u(x, y)}{\partial y}\right)-\left(\lambda_{y}+s_{y} y\right)\left(\frac{\partial v(x, y)}{\partial y}\right) \\
& =-C_{r} \frac{\partial r(x, y)}{\partial y}+\frac{\left(C_{t}+C_{f} f+C_{r} r(x, y)\right) \cos \theta}{V+u(x, y) \cos \theta+v(x, y) \sin \theta}\left(\frac{\partial u(x, y)}{\partial y}\right)+\frac{\left(C_{t}+C_{f} f+C_{r} r(x, y)\right) \sin \theta}{V+u(x, y) \cos \theta+v(x, y) \sin \theta}\left(\frac{\partial v(x, y)}{\partial y}\right) .
\end{aligned}
$$

Splitting the right hand side of Eq. (A2) into two parts to get

$$
\begin{aligned}
& \frac{(V+u(x, y) \cos \theta+v(x, y) \sin \theta)\left[-\left(\lambda_{y}+s_{y} \dot{y}\right) \cos \theta\right]}{\left(C_{t}+C_{f} f+C_{r} r(x, y)\right)} \\
& \quad=\left[C_{r} \frac{\partial r(x, y)}{\partial y}\right] \frac{\cos \theta(V+u(x, y) \cos \theta+v(x, y) \sin \theta)}{\left(C_{t}+C_{f} f+C_{r} r(x, y)\right)}-\cos ^{2} \theta\left(\frac{\partial u(x, y)}{\partial y}\right)-\cos \theta \sin \theta\left(\frac{\partial v(x, y)}{\partial y}\right)
\end{aligned}
$$




$$
\begin{aligned}
& \frac{(V+u(x, y) \cos \theta+v(x, y) \sin \theta)\left[\left(\lambda_{x}+s_{x} \dot{x}\right) \sin \theta\right]}{\left(C_{t}+C_{f} f+C_{r} r(x, y)\right)} \\
& \quad=\left[-C_{r} \frac{\partial r(x, y)}{\partial x}\right] \frac{\sin \theta(V+u(x, y) \cos \theta+v(x, y) \sin \theta)}{\left(C_{t}+C_{f} f+C_{r} r(x, y)\right)}+\sin \theta \cos \theta\left(\frac{\partial u(x, y)}{\partial x}\right)+\sin ^{2} \theta\left(\frac{\partial v(x, y)}{\partial x}\right) .
\end{aligned}
$$

Summing Eq. (A3) and Eq. (A4) to obtain dynamical equation for the optimal heading,

$$
\begin{aligned}
\dot{\theta}=\frac{(V+u(x, y) \cos \theta+v(x, y) \sin \theta)}{\left(C_{t}+C_{f} f+C_{r} r(x, y)\right)} & {\left[-C_{r} \sin \theta \frac{\partial r(x, y)}{\partial x}+C_{r} \cos \theta \frac{\partial r(x, y)}{\partial y}\right] } \\
+ & \sin ^{2} \theta\left(\frac{\partial v(x, y)}{\partial x}\right)+\sin \theta \cos \theta\left(\frac{\partial u(x, y)}{\partial x}-\frac{\partial v(x, y)}{\partial y}\right)-\cos ^{2} \theta\left(\frac{\partial u(x, y)}{\partial y}\right) .
\end{aligned}
$$

When $C_{r}$ is zero, Eq. (A5) is reduced to solution in the Zermelo problem.

\section{References}

${ }^{1}$ Schumann, U., 1996: On conditions for contrail formation from aircraft exhausts. Meteor. Z., N. F. 5, 3-22.

${ }^{2}$ Duda, D.P., Minnis, P., Costulis, P.K., and Palikonda, R. "CONUS Contrail Frequency Estimated from RUC and Flight Track Data," European Conference on Aviation, Atmosphere, and Climate, Friedrichschafen at Lake Constance, Germany, JuneJuly, 2003.

${ }^{3}$ Waitz, I., Townsend, J., Cutcher-Gershenfeld, J., Greitzer, E., and Kerrebrock, J. Report to the United States Congress:

Aviation and the Environment, A National Vision, Framework for Goals and Recommended Actions. Partnership for Air Transportation Noise and Emissions Reduction, MIT, Cambridge, MA, 2004.

${ }^{4}$ Klima, K., "Assessment of a Global Contrail Modeling Method and Operational Strategies for Contrail Mitigation,” M.S. Thesis, MIT, 2005.

${ }^{5}$ Mannstein, H., Spichtinger, P., and Gierens, K., “A Note on How to Avoid Contrail Cirrus,” Transportation Research Part $D$, Vol. 10, No. 5, 2005, pp. 421-426.

${ }^{6}$ Campbell S.E., Neogi, N. A., and Bragg, M. B., "An Optimal Strategy for Persistent Contrail Avoidance,” AIAA 20086515, AIAA Guidance, Navigation, and Control Conference, Honolulu, Hawaii, 2008.

${ }^{7}$ Gierens K., Lim, L. and Eleftheratos K. "A Review of Various Strategies for Contrail Avoidance," The Open Atmosphere Science Journal, 2, 1-7, 2008.

${ }^{8}$ Naidu, D. S., and Calise, A. J., "Singular Perturbations and Time Scales in Guidance and Control of Aerospace Systems: A Survey," AIAA Journal of Guidance, Control and Dynamics, Vol. 24, No. 6, pp. 1057-1078, Nov.-Dec., 2001.

${ }^{9}$ Bryson, A. E., and Ho, Y. C., Applied Optimal Control, Taylor and Francis, Levittown, PA, 1975.

${ }^{10}$ Appleman, H., 1953: The formation of exhaust condensation trails by jet aircraft. Bull. Amer. Meteor. Soc., Vol. 34, 14-20.

${ }^{11}$ Degrand J. Q., Carleton, A. M., Travis D. J., and Lamb, P. J., "A Satellite-Based Climate Description of Jet Aircraft Contrails and Associations with Atmospheric Conditions, 1977-79," Journal of Applied Meteorology, Vol. 39, pp. 1434-1459.

${ }^{12}$ Palikonda R., Minnis P., Duda, D.P., and Mannstein H., "Contrail coverage derived from 2001 AVHRR data over the continental United States of America and surrounding areas," Meteorologische Zeitschrift, Vol. 14, No. 4, 525-536, August 2005.

${ }^{13}$ Alduchov, O. A., and R.E. Eskridge, "Improved Magnus form Approximation of Saturation Vapor Pressure," Journal of Applied Meteorology, Vol. 35, 601-609.

${ }^{14}$ Calise, A. J. and Moerder, D. D., "Singular Perturbation Techniques for Real Time Aircraft Trajectory Optimization and Control," NASA CR-3597, 1982.

${ }^{15}$ Charavarity, A. J. M., "Application of Singular Perturbation Theory to Onboard Aircraft Trajectory Optimization," Boeing Commercial Airplane Company, Seattle, WA, Document D6-51308TN 1983.

${ }^{16}$ Vian, J. L., and Moore, J. R., “Trajectory Optimization with risk Minimization for Military Aircraft,” J. Guidance, Vol. 12, No., 3, May-June 1989.

${ }^{17}$ Burrows, J. W., "Fuel-Optimal Aircraft Trajectories with Fixed Arrival Times," AIAA Journal of Guidance, Control., and Dynamics, Vol. 6, Jan.-Feb. 1983, pp. 14-19.

${ }^{18}$ Press, William H., Teukolsky, Saul A., Vetterling, William T., and Flannery, Brian P., Numerical Recipes in C: The Art of Scientific Computing, $2^{\text {nd }}$ ed., Cambridge University Press, Cambridge, England, 1992, Chapter 17.

${ }^{19}$ Eurocontrol Experimental Center (EEC). "User Manual for the Base of Aircraft Data (BADA), Revision 3.6." EEC Note No. 10/04. Project ACE-C-E2. September 2004.

${ }^{20}$ CDM/DRVSM Work Group Report, "Benefit Analysis and Report for Domestic Reduced Vertical Separation Minimum (DRVSM),” FAA Air Traffic Organization System Operations Services, September 2005. 